\title{
Table of government and regulatory body reports, codes, rules and publications
}

\section{Australian Securities Exchange (ASX) (AUSTRALIA)}

\begin{tabular}{|l|l|}
\hline Report, Code, Rule or Publication & Page \\
\hline ASX 2003 Best Practice Recommendations & \\
The original ASX best practice recommendations were & \\
contained in the ASX Corporate Governance Council, & \\
Principles of Good Corporate Governance and Best Practice & $52,54,96$, \\
Recommendations, Australian Securities Exchange, March & $151-152$, \\
2003 ('ASX 2003 Best Practice Recommendations'). & $176-182$ \\
& \\
& \\
\hline $\begin{array}{l}\text { ASX Draft Recommendations } \\
\text { Proposed changes to the ASX 2003 Best Practice }\end{array}$ & \\
Recommendations were the subject of public comment. See & \\
ASX Corporate Governance Council, Principles of Good \\
Corporate Governance and Best Practice Recommendations, \\
Exposure Draft of Changes, Australian Securities Exchange, \\
2 November 2006 ('ASX Draft Recommendations'). \\
\hline
\end{tabular}




\begin{tabular}{|c|c|}
\hline Report, Code, Rule or Publication & Page \\
\hline $\begin{array}{l}\text { ASX 2007-10 Revised Principles } \\
\text { The ASX } 2003 \text { Best Practice Recommendations were revised } \\
\text { on } 2 \text { August 2007. See ASX Corporate Governance Council, } \\
\text { Corporate Governance Principles and Recommendations, } \\
\text { Second Edition, Australian Securities Exchange, } 2 \text { August } \\
\text { 2007. These Principles and Recommendations were further } \\
\text { amended in June 2010. See ASX Corporate Governance } \\
\text { Council: } \\
\text { - Corporate Governance Principles and Recommendations } \\
\text { with 2010 Amendments, June } 2010 \text { ('ASX 2007-10 } \\
\text { Revised Principles'); } \\
\text { - Revised Corporate Governance Principles and } \\
\text { Recommendations (Second Edition, August } 2007 \text { ); } \\
\text { - Marked-Up Amendments dated } 30 \text { June 2010 to the } \\
\text { Second Edition of the Corporate Governance Principles } \\
\text { and Recommendations ('ASX } 2010 \text { Marked-Up } \\
\text { Amendments'); and } \\
\text { - Summary Table of the } 30 \text { June Changes to the Second } \\
\text { Edition of the Corporate Governance Principles and } \\
\text { Recommendations ('ASX } 2010 \text { Summary Table'). } \\
\text { These documents are available at http://www.asxgroup.com. } \\
\text { au/corporate-governance-council.htm (accessed } 6 \text { March } \\
\text { 2015). }\end{array}$ & $\begin{array}{l}52,54,56,58, \\
96-98,151,153, \\
176-182,326 \\
52,54,56,58, \\
96-98,151,153, \\
176-182,326 \\
153 \\
153,182 \\
153,182\end{array}$ \\
\hline $\begin{array}{l}\text { ASX } 2014 \text { Principles and Recommendations } \\
\text { The ASX 2007-10 Revised Principles were revised and } \\
\text { replaced by the Third Edition of the Principles and } \\
\text { Recommendations which were released on } 27 \text { March } 2014 \\
\text { and (at p 7) took effect for a company's financial year } \\
\text { commencing on or after } 1 \text { July } 2014 \text { ('ASX } 2014 \text { Principles } \\
\text { and Recommendations'). } \\
\text { For a comparison of the ASX 2007-10 Revised Principles } \\
\text { and the ASX } 2014 \text { Principles and Recommendations, } \\
\text { see Translation Table, Corporate Governance Council } \\
\text { Recommendations (New to Old) and (Old to New), ('CGC } \\
\text { Recommendations Translation Tables'). } \\
\text { These documents are available at http://www.asx.com.au/ } \\
\text { regulation/corporate- governance-council.htm (accessed } 6 \\
\text { March 2015). }\end{array}$ & $\begin{array}{l}38,40,42,52, \\
54,56,60, \\
97-99,102,151, \\
153,176-183, \\
253,323,326, \\
330\end{array}$ \\
\hline
\end{tabular}




\begin{tabular}{|c|l|}
\hline Report, Code, Rule or Publication & Page \\
\hline $\begin{array}{l}\text { Australian Securities Exchange, ASX Listing Rules, General } \\
\text { Rule 3.1, Continuous Disclosure, 01/01/2003, p 301, } \\
\text { available at http://www.asx.com.au/ListingRules/ } \\
\text { chapters/Chapter03.pdf, p 302, (accessed 02/02/09) }\end{array}$ & \\
\hline $\begin{array}{l}\text { Australian Securities Exchange, ASX Listing Rules, } \\
\text { Rule 4.10.3, available at http://www.asx.com.au/ } \\
\text { ListingRules/chapters/Chapter04.pdf (accessed } \\
\text { 26/08/10) }\end{array}$ & 323 \\
\hline
\end{tabular}

\section{Financial Reporting Council (UK)}

\begin{tabular}{|l|l|}
\hline Report, Code, Rule or Publication & Page \\
\hline $\begin{array}{c}\text { Financial Reporting Council (FRC), The Combined Code } \\
\text { on Corporate Governance, June 2006, London ('UK } \\
\text { Combined Code') }\end{array}$ & $\begin{array}{l}151-152, \\
171-175,226\end{array}$ \\
\hline $\begin{array}{l}\text { Financial Reporting Council (FRC), The UK Approach } \\
\text { to Corporate Governance, November 2006, London } \\
\text { ('FRC UK Governance Approach 2006') }\end{array}$ & 146,171 \\
\hline $\begin{array}{l}\text { Financial Reporting Council (FRC), The UK Approach to } \\
\text { Corporate Governance, October 2010, London ('FRC } \\
\text { UK Governance Approach 2010') }\end{array}$ & 146 \\
\hline $\begin{array}{l}\text { Financial Reporting Council (FRC), The UK Corporate } \\
\text { Governance Code, June 2010, as amended in } \\
\text { September 2012, London ('UK Corporate } \\
\text { Governance Code 2010-12') }\end{array}$ & $151-152$, \\
\hline
\end{tabular}




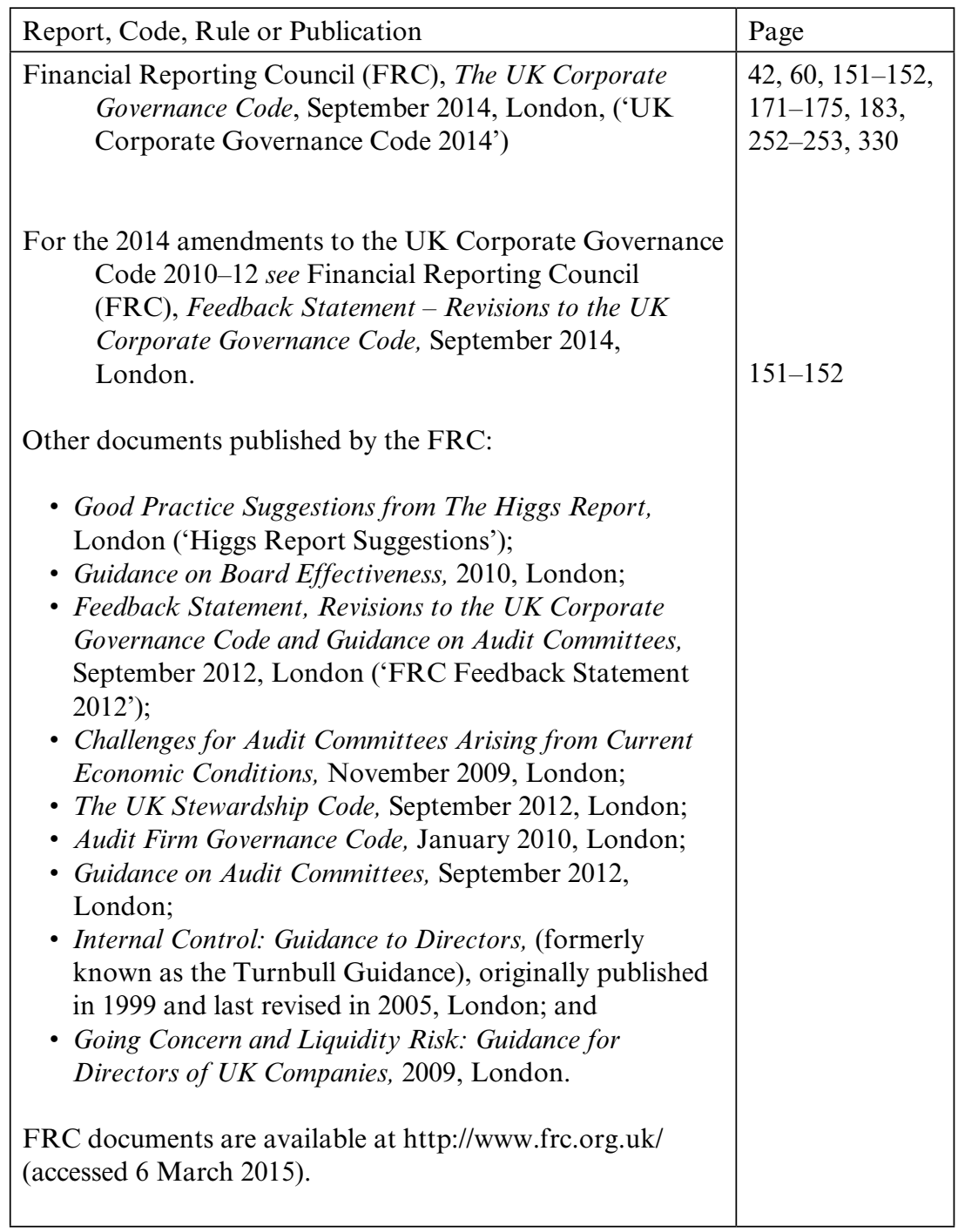




\section{Other Government And Regulatory Bodies}

\begin{tabular}{|c|c|}
\hline Report, Code, Rule or Publication & Page \\
\hline $\begin{array}{l}\text { Australian Government, Corporations and Markets } \\
\text { Advisory Committee (CAMAC), Executive } \\
\text { Remuneration, Report, April 2011, available at http:// } \\
\text { www.camac.gov.au/camac/camac.nsf/byHeadline/ } \\
\text { PDFFinal+Reports+2011/\$file/Executive_ } \\
\text { remuneration_report_April11.pdf (accessed } 6 \text { March } \\
\text { 2015) }\end{array}$ & 300 \\
\hline $\begin{array}{l}\text { Australian Government, Corporations and Markets } \\
\text { Advisory Committee, The Social Responsibility } \\
\text { of Corporations, Report, December 2006, Sydney, } \\
\text { available at http://www.camac.gov.au (accessed } \\
6 \text { March 2015), ('CAMAC Social Responsibility } \\
\text { Report') }\end{array}$ & $93-95,100-101$ \\
\hline $\begin{array}{l}\text { Australian Government, Productivity Commission, } \\
\text { Executive Remuneration in Australia, Productivity } \\
\text { Commission Inquiry Report No 49, } 19 \text { December } \\
\text { 2009, available at http://www.pc.gov.au/_data/assets/ } \\
\text { pdf_file/0008/93590/executive-remuneration-report. } \\
\text { pdf, (accessed } 2 \text { January 2013) }\end{array}$ & 300 \\
\hline $\begin{array}{l}\text { Business Roundtable, Principles of Corporate Governance } \\
\text { 2005, A White Paper by Business Roundtable, } \\
\text { November 2005, Washington, DC ('Business } \\
\text { Roundtable Principles 2005') } \\
\text { The } 2005 \text { Principles were updated in April } 1010 \text { and } \\
\text { June 2012. For the current Principles, see Business } \\
\text { Roundtable, Principles of Corporate Governance } \\
\text { 2012, June 2012, Washington, DC ('Business } \\
\text { Roundtable Principles 2012') available at http:// } \\
\text { businessroundtable.org/uploads/studies-reports/ } \\
\text { downloads/BRT_Principles_of_Corporate_ } \\
\text { Governance_-2012_Formatted_Final.pdf (accessed } 27 \\
\text { March 2013) }\end{array}$ & $\begin{array}{l}151-152 \\
165-168\end{array}$ \\
\hline
\end{tabular}




\begin{tabular}{|c|c|}
\hline Report, Code, Rule or Publication & Page \\
\hline $\begin{array}{l}\text { California Public Employees' Retirement System } \\
\text { (CalPERS), Global Principles of Accountable } \\
\text { Corporate Governance, Sacramento, California, } \\
\text { available at http://www.calpers-governance.org/ } \\
\text { docs-sof/principles/2011-11-14-global-principles-of- } \\
\text { accountable-corp-gov.pdf, updated } 14 \text { November } \\
2011 \text { (accessed } 14 \text { August 2013). For the current } \\
\text { Global Principles, see California Public Employees' } \\
\text { Retirement System (CalPERS), Global Principles of } \\
\text { Accountable Corporate Governance, } 19 \text { May 2014, } \\
\text { Sacramento, California, available at http://www. } \\
\text { calpers-governance.org/docs-sof/principles/2014- } \\
\text { 05-calpers-global-principles-accountable-corp-gov. } \\
\text { pdf. (accessed 13 March 2015), ('CalPERS Global } \\
\text { Governance Principles 2014') }\end{array}$ & $\begin{array}{l}151-152 \\
165-168\end{array}$ \\
\hline $\begin{array}{l}\text { The Committee on the Financial Aspects of Corporate } \\
\text { Governance, Report of the Committee on the Financial } \\
\text { Aspects of Corporate Governance, } 1 \text { December 1992, } \\
\text { London: Gee \& Co Ltd ('Cadbury Report') }\end{array}$ & $\begin{array}{l}3,28,151-152 \\
171-175,326\end{array}$ \\
\hline $\begin{array}{l}\text { Commonwealth Association for Corporate Governance, } \\
\text { Principles for Corporate Governance in the } \\
\text { Commonwealth, Towards Global Competitiveness and } \\
\text { Economic Accountability, Final Version, November } \\
\text { 1999, ('CACG Guidelines'), available at http://www. } \\
\text { ecseonline.com/PDF/CACG\%20Guidelines } \% 20-\% 20 \\
\text { Principles } \% 20 \text { for } \% 20 \text { Corporate } \% 20 \text { Governance } \% 20 \\
\text { in } \% 20 \text { the } \% 20 \text { Commonwealth.pdf (accessed } 6 \text { March } \\
\text { 2015) }\end{array}$ & $\begin{array}{l}6-27,60,151 \\
154-157,359 \\
361\end{array}$ \\
\hline $\begin{array}{l}\text { The Conference Board, Inc., Commission on Public Trust } \\
\text { and Private Enterprise, 9 January 2003, available } \\
\text { at http://www.conference-board.org/publications } \\
\text { (accessed } 6 \text { March 2015), Part 2: 'Corporate } \\
\text { Governance: Principles, Recommendations and } \\
\text { Specific Best Practice Suggestions', ('Conference } \\
\text { Board Principles') }\end{array}$ & $\begin{array}{l}151-152 \\
165-168\end{array}$ \\
\hline
\end{tabular}




\begin{tabular}{|c|c|}
\hline Report, Code, Rule or Publication & Page \\
\hline $\begin{array}{l}\text { The International Bank for Reconstruction and } \\
\text { Development/The World Bank and Organisation } \\
\text { for Economic Co-operation and Development, } \\
\text { Global Corporate Governance Forum: } 1 \text { Focus: } \\
\text { Corporate Governance and Development, Stijn } \\
\text { Claessens (author), Washington, DC,2003. See also } \\
\text { The International Bank for Reconstruction and } \\
\text { Development/The World Bank and Organisation For } \\
\text { Economic Co-operation and Development, Global } \\
\text { Corporate Governance Forum: } 2 \text { Toolkit: Developing } \\
\text { Corporate Governance Codes of Best Practice, User } \\
\text { Guide: Volume 1 Rationale, Washington, DC 2005 } \\
\text { ('WB-OECD Global Corporate Governance Forum') }\end{array}$ & 26 \\
\hline $\begin{array}{l}\text { International Corporate Governance Network, ICGN } \\
\text { Statement on Global Corporate Governance Principles, } \\
\text { July 8, 2005, ICGN Annual Conference, London, } \\
\text { ('ICGN Statement'). The Principles in the ICGN } \\
\text { Statement were updated in } 2009 \text { and appear as Part } \\
\text { C International Principles of Accountable Corporate } \\
\text { Governance of the CalPERS Global Governance } \\
\text { Principles } 2014 \text { above available at http://www.calpers- } \\
\text { governance.org/docs-sof/principles/2014-05-calpers- } \\
\text { global-principles-accountable-corp-gov.pdf. (accessed } \\
13 \text { March 2015) }\end{array}$ & $\begin{array}{l}60,151, \\
154-157,359, \\
361\end{array}$ \\
\hline $\begin{array}{l}\text { Investment \& Financial Services Association Limited } \\
\text { (IFSA), Blue Book, IFSA Guidance Note No. } \\
\text { 2.00, Corporate Governance: A Guide for Fund } \\
\text { Managers and Corporations, 6th edn, June 2009, } \\
\text { available at, http://www.fsc.org.au/downloads/ } \\
\text { file/IFSAGuidanceNotes/2GN_2_Corporate_ } \\
\text { Governance_2009.pdf ('IFSA Blue Book') (accessed } 6 \\
\text { March 2015) }\end{array}$ & $\begin{array}{l}151,153 \\
176-182\end{array}$ \\
\hline
\end{tabular}




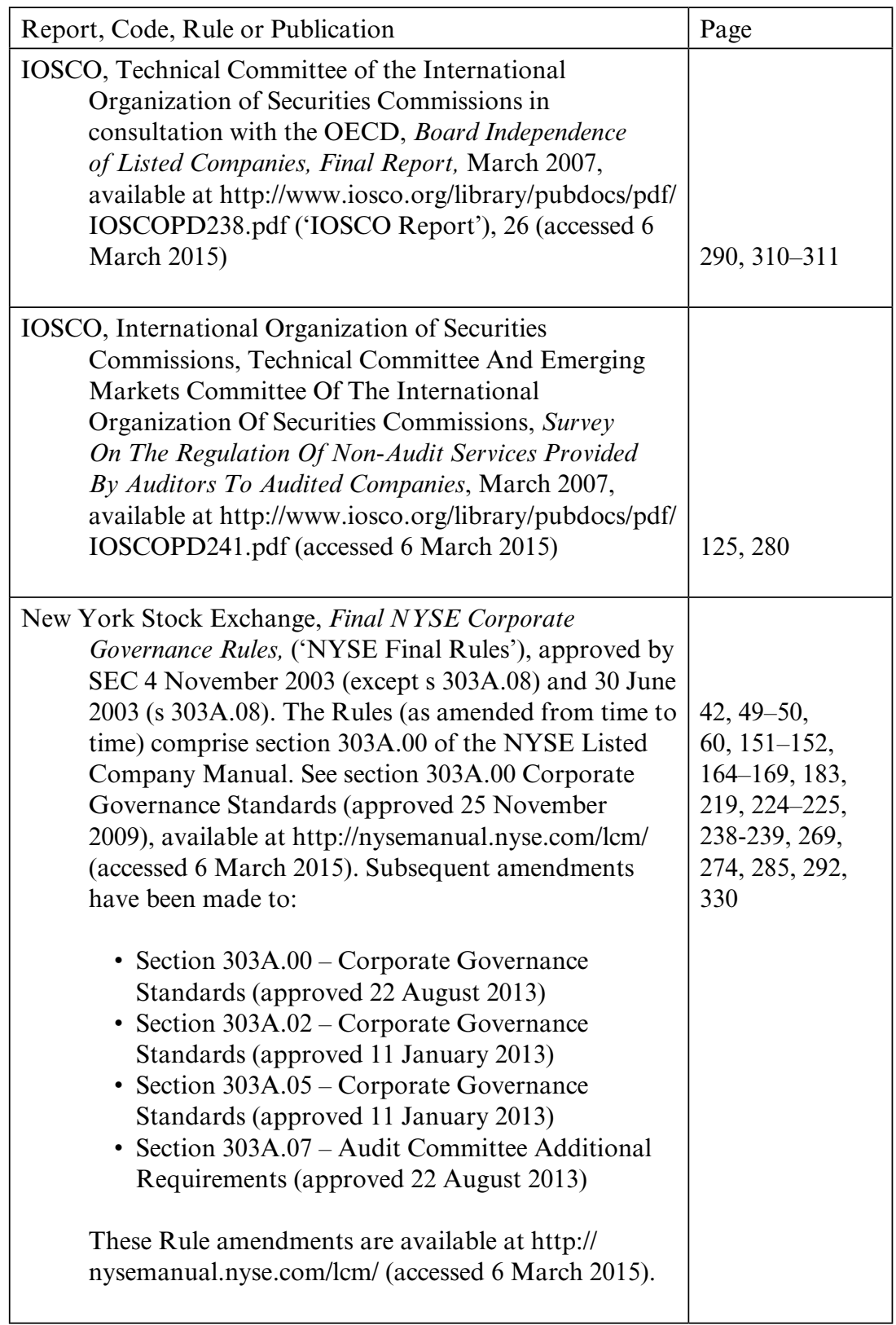




\begin{tabular}{|l|l|}
\hline Report, Code, Rule or Publication & Page \\
\hline Organisation for Economic Co-Operation and & $4,26,28,38$, \\
Development (OECD), OECD Principles of & $40,42,52-53$, \\
$\begin{array}{l}\text { Corporate Governance 2004, 2004, OECD } \\
\text { Publications Service, Paris ('OECD Principles') }\end{array}$ & $60,99,151$, \\
see http://www.oecd.org/document/49/0,3343 & $153-158,165-$ \\
,en_2649_34813_31530865_1_1_1_37439,00.html & $169,172-175$, \\
(accessed 6 March 2015). & $177-181,261-$ \\
& $262,326-327$, \\
& $329-330,359$, \\
\hline $\begin{array}{l}\text { Ramsay, Ian, Independence of Australian Company Auditors: } \\
\text { Review of Current Australian Requirements and } \\
\text { Proposals for Reform, Report to the Minister for } \\
\text { Financial Services and Regulation, Commonwealth } \\
\text { of Australia, October 2001 (as printed in March } \\
\text { 2002), available at http://archive.treasury.gov.au/ } \\
\text { contentitem.asp?ContentID=296 (accessed 6 March } \\
\text { 2015) }\end{array}$ & \\
\hline $\begin{array}{l}\text { Treadway Commission, Report of the National Commission } \\
\text { on Fraudulent Financial Reporting, National } \\
\text { Commission on Financial Reporting (US), 1987. }\end{array}$ & 282 \\
\hline
\end{tabular}

\section{Administrators' Report}

\begin{tabular}{|l|l|}
\hline Report & Page \\
\hline PPB Advisory, Hastie Group Limited and Specific & $2,10-11,37-38$, \\
Subsidiaries, Report by Joint and Several & $40,42-43,56-57$, \\
Administrators Pursuant to Section 439A of & $107,109-111$, \\
the Corporations Act 2001, 21 January 2013, & $113,115-118$, \\
available at http://www.ppbadvisory.com/creditor- & $122-123$, \\
information/v/281/hastie-group/2nd-creditor-meeting & $128-130,132$, \\
('Administrators' Report'), (accessed 6 March 2015) & 138,361 \\
\hline
\end{tabular}


Francesco de Zwart - 9781784715526

Downloaded from PubFactory at 04/26/2023 02:11:57PM via free access 